\title{
Classification of Japanese Documents and Ranking of Representative Documents by Using the Characteristic of the Frequencies of Words
}

\author{
Jun Kimura \\ JustSystems Corp., 6-8-1 Nishinjuku, Shinjuku-ku, Tokyo 163-6017 \\ Yasunari Yoshitomi, Masayoshi Tabuse \\ Graduate School of Life and Environmental Sciences, Kyoto Prefectural University, \\ 1-5 Nakaragi-cho, Shimogamo, Sakyo-ku, Kyoto 606-8522, Japan \\ E-mail: \{yoshitomi, tabuse\}@kpu.ac.jp \\ http://www2.kpu.ac.jp/ningen/infsys/English_index.html
}

\begin{abstract}
We developed a method for classification of Japanese documents and ranking of representative documents by using the characteristic of the frequencies of nouns. A representative document is defined as a document whose feature vector is the closest to the center of gravity of the class in the feature vector space among all documents belonging to the class belonging to the class. The ranking of representative documents is decided in descending order of the number of documents belonging to the class.
\end{abstract}

Keywords: Clustering, Document classification, Extraction of representative document, Frequency of nouns.

\section{Introduction}

The number of Web pages on the Internet continues to increase. Consequently, it is very difficult to read through all of the Web pages of interest to us, especially because there are too many similar Web pages. For efficiently acquiring useful Web pages, it is necessary to select only Web pages having important and independent contents so that we can acquire the essential information.

A Web page includes various kinds of media, such as documents, images, and sounds. We focus on selecting a Web page on the Internet according to the characteristics of the document on the page. The classification of documents has received considerable attention in document analysis research. ${ }^{1-8}$ However, to the best of our knowledge, no research has investigated the selection of a representative document in a class of documents, followed by the ranking of several representative documents in order of importance or useful meaning for the user.

In the present study, we developed a method for classification of Japanese documents and ranking of representative documents by using the characteristic of the frequencies of nouns.

\section{Proposed Method}

\subsection{Extraction of nouns}

First, all nouns in a document are extracted by $\mathrm{MeCab}^{9}$, which resolves the document into several morphemes (Fig. 1). 


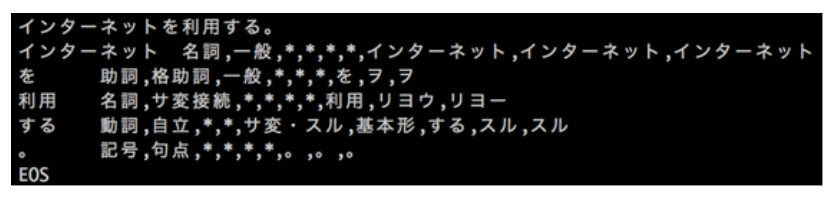

Fig. 1. Output of MeCab.

\subsection{Connection of nouns having a meaning as a set}

Some nouns directly connecting each other are treated as one noun in the case that they have a meaning by assuming one noun. For example, 2014 and 年 in Japanese have a meaning as a set of 2014 年, where 年 in Japanese means year in English.

\subsection{Addition of negative attribution}

A noun can have either a positive or negative attribution. When a sentence expresses a negative meaning with the use of "not", the extracted nouns in the sentence are treated as having a negative attribution. The noun having a negative attribution is treated as being different from the noun with a positive attribution when making a feature vector for the document containing the noun.

\subsection{Feature vector generation}

After every noun composed of 1) only one hiragana character (rounded Japanese phonetic syllabary), 2) only one katakana character (angular Japanese syllabary), or 3) a symbol is erased, a feature vector having the relative frequency of each noun as an element is generated for each document. The relative frequency is defined as the ratio of the frequency of the noun to that of all nouns in the document, except nouns erased by the above criterion.

\subsection{Document classification and extraction of representative document in each class}

For clustering, we use the Ward method. The representative document is defined as the document whose feature vector is the closest to the center of gravity of the class in the feature vector space among all documents belonging to the class.

\subsection{Ranking of representative documents}

The first-rank document is defined as the document whose feature vector is the closest to the center of gravity of all documents in the feature vector space. In this case, the number of classes is one. Afterward, the number of classes is increased in increments of one, and then the ranking from the second rank for the representative documents is decided in descending order of the number of documents belonging to the class for each number of classes. The maximum number $J$ of classes in stepwise clustering is given beforehand. Although a document can be selected more than once in the ranking process, only the first selection of the document is accepted.

\section{Calculation Environment}

The development of the system and the experiments for evaluation of the proposed method were performed on a personal computer: DELL OPTIPLEX 780 (CPU: Intel Core2 Duo CPU E8400 3.00 GHz, RAM: 4.00 GB), OS: Microsoft Windows 7 Professional, Development language: Python 2.7.3.

\section{Experiments and Discussion}

\subsection{Document classification}

First, we evaluated the performance of document classification by the proposed method. We gathered 20 documents on politics (document nos. 1-10) and horse racing (document nos. 11-20) from Yahoo! Japan News $^{10}$ in January 2013, and then the number of clusters was set to two. The output of our system is shown in Table 1. The clusters of $\mathrm{C}_{1}$ and $\mathrm{C}_{2}$ were composed of the documents on politics and horse racing, respectively. As shown in the table, document classification by the proposed method was completely accurate.

Table 1. Nos. of documents belonging to each cluster.

\begin{tabular}{ll}
\hline Cluster $\mathrm{C}_{1}$ & ${\text { Cluster } \mathrm{C}_{2}}$ \\
\hline $1,2,3,4,5$, & $11,12,13,14,15$, \\
$6,7,8,9,10$ & $16,17,18,19,20$ \\
\hline
\end{tabular}

\subsection{Extraction of representative documents}

\subsubsection{Experiment I}

Next, we evaluated the performance of extraction of the representative document by the proposed method. We gathered the top 20 documents retrieved from Google News ${ }^{11}$ and those from Yahoo! Japan News by using the keyword '大阪府高校' in Japanese, which means Osaka Prefecture High School in English, on 22 January 2013. The name of a document obtained was set to be the same as the rank of each retrieval, and then all documents were categorized. 
The name of a category was decided to be the content name when more than two documents had similar content, and otherwise the document was assigned to the category of "Others". The categorization was manually performed through our understanding of each document, while the clustering was performed by the proposed method. Therefore, it was not guaranteed that the clustering result would correspond with the document group structure given by the manual categorization.

\section{(a) Google News}

Table 2 shows the document group structure when we used Google News in our experiment. The number of kinds of categories was five. Table 3 shows the ranking of representative documents given by the proposed method for $J=4$.

Table 2. Document group structure I.

\begin{tabular}{llll}
\hline Category & Rugby & \multicolumn{3}{l}{ Board of education } \\
\hline Document No. & $1,9,12,18,20$ & $2,4,5,11,16,17,19$ \\
\hline Category & Skating & Distress accident & Others \\
\hline Document No. & 3,14 & $6,7,8,15$ & 10,13 \\
\hline
\end{tabular}

Table 3. Result I.

Ranking of representative documents expressed by Nos.
$6,1,4,3$

As shown in Table 3, the four representative documents were successfully extracted one by one from all categories, except the category of "Others", in the order of "Distress accident", "Rugby", "Board of Education", and "Skating".

(b) Yahoo! Japan News

Table 4 shows the document group structure when we used Yahoo! Japan News in our experiment. The number of kinds of categories was five. Table 5 shows the ranking of representative documents given by the proposed method for $J=4$.

As shown in Table 5, the four representative documents were successfully extracted one by one from all categories, except the category of "Rugby", in the order of "Board of Education", "Center exam", "Distress accident", and "Others".

Table 4. Document group structure II.

\begin{tabular}{llll}
\hline Category & Rugby & \multicolumn{2}{c}{ Board of education } \\
\hline Document No. & 15,19 & $2,3,4,11,14,16,17,18,20$ \\
\hline Category & Distress accident & Center exam. & Others \\
\hline Document No. & $8,12,13$ & 9,10 & $1,5,6,7$ \\
\hline
\end{tabular}

Table 5. Result II. Ranking of representative documents expressed by Nos. $18,10,8,5$

\subsubsection{Experiment II}

We gathered the top 20 documents obtained by the retrievals from Google News and Yahoo! Japan News by using the retrieval keyword of "Microsoft" on 22 January 2013. The name of a document obtained was set to be the same as the rank of each retrieval, and then all documents were categorized in the same manner described in Section 4.2.1.

\section{(a) Google News}

Table 6 shows the document group structure when we used Google News in our experiment. The number of kinds of categories was four. Table 7 shows the ranking of representative documents given by the proposed method for $J=4$. As shown in Table 7, the six representative documents were extracted from all categories in the order of "Others", "Others", "Windows 8", "MS Essentials", "Surface", and "Others".

Table 6. Document group structure III.

\begin{tabular}{lll}
\hline Category & Windows 8 & MS Essentials \\
\hline Document No. & $3,5,14$ & $2,6,12$ \\
\hline Category & Surface & Others \\
\hline Document No. & $9,11,15$ & $1,4,7,8,10,13$, \\
& & $16,17,18,19,20$ \\
\hline
\end{tabular}

Table 7. Result III.

Ranking of representative documents expressed by Nos. $18,4,3,6,11,20$

(b) Yahoo! Japan News

Table 8 shows two kinds of categories. Table 9 shows the ranking for the document groups shown in Table 8 .

Table 8. Document group structure IV.

\begin{tabular}{lll}
\hline Category & Cannon ITS & Others \\
\hline Document No. & $6,9,14$ & $1,2,3,4,5,7,8,10,11,12$, \\
& & $13,15,16,17,18,19,20$ \\
\hline
\end{tabular}

Table 9. Result IV.

Ranking of representative documents expressed by Nos. $2,9,15,13,20,11$

As shown in Table 9, the six documents were extracted in the order of categories of "Others", "Cannon ITS", and four sets of "Others". In this 
document group, almost all documents belonged to the category of "Others". However, one document was extracted from the category of "Cannon ITS" in the second order.

\subsection{Discussion}

\subsubsection{Document group structure dependency}

When the document group had a distinct structure such as that in Sections 4.2.1 (a) \& (b) and 4.2.2 (a), the performance of the proposed method was almost completely accurate in that the documents could be extracted one by one from all categories except "Others". On the other hand, when the document group had a scattered structure such as that in Section 4.2.2 (b), it might not be meaningful to try to retrieve almost all contents by extracting the representative documents by the proposed method.

\subsubsection{Performance improvement}

It might be necessary to apply the proposed method to many document groups to find assignments of the proposed method. It also might be effective to use a thesaurus for reducing the dimension of the feature vector space to potentially extract more representative documents and/or to reduce the calculation cost.

4.3.3. Definition of representative document

In the present study, the representative document was geometrically defined in the feature vector space. It is necessary to investigate the validity of the definition through questionnaires. In the investigation, other definitions on the representative document might warrant further discussion.

\section{Conclusion}

We developed a method for classification of Japanese documents and ranking of representative documents by using the characteristic of the frequencies of nouns. Experiments of collecting Web pages for evaluating the efficiency of the proposed method proved its usefulness.

\section{References}

1. F. Can and E. A. Ozkarahan, Computation of term/document discrimination values by use of the cover coefficient concept, J. Amer. Soc. for Inf. Sci., 38(3) (1987) 171-183.

2. A. Kawai, An automatic document classification method based on a semantic category frequency analysis (in Japanese), J. IPSJ 33(9) (1992) 1114-1122.

3. N. Yuasa, T. Ueda, and F. Togawa, Classifying articles using lexical co-occurrence in large document databases (in Japanese), J. IPSJ 36(8) (1995) 1819-1827.

4. K. Hatano, R. Sano, Y. Duan, and K. Tanaka, A classification view mechanism for web documents based on self-organizing maps and search engines (in Japanese), J. IPSJ 40(SIG 3(TOD1)) (1999) 47-59.

5. H. Takamura and Y. Matsumoto, Co-clustering for text categorization (in Japanese), J. IPSJ 44(2) (2003) 443450.

6. H. Takamura and Y. Matsumoto, Constructive induction and text categorization with SVMs (in Japanese), J. IPSJ 44(SIG 3(TOD 17)) (2003) 1-10.

7. R. Kusaya, T. Yamamura, H. Kudo, T. Matsumoto, Y. Takeuchi, and N. Ohnishi, Measuring similarity between documents using term frequency (in Japanese), Trans IEICE J87-D- II (2) (2004) 661-672.

8. Y. Bamba, K. Shinzato, T. Shibata, and S. Kurohashi, Web information observation using keyword distillation based clustering (in Japanese), J. IPSJ 50(4) (2009) 1399-1409.

9. MeCab, http://mecab.sourceforge.net/ Accessed 1 November 2012.

10. Yahoo! JAPAN News, http://headlines.yahoo.co.jp/hl Accessed 22 January 2013.

11. Google News, https://news.google.co.jp/ Accessed 22 January 2013. 